\title{
Design of a Cyber Physical Industrial Robotic Manipulator
}

\author{
Emmanuel Francalanza*, Alec Fenech and Paul Cutajar \\ Department of Industrial and Manufacturing Engineering, University of Malta, Malta
}

Submission: February 05, 2018; Published: April 10, 2018

*Corresponding author: Emmanuel Francalanza, CERU, Department of Industrial and Manufacturing Engineering, University of Malta, Msida, MSD2080, Malta, Tel: +356-2340-2060; E-mail address: emmanuel.francalanza@um.edu.mt

Abstract

A key component of Cyber Physical Production Systems (CPPS) are connected Cyber Physical Machines (CPMs), or smart machines, which can act independently to each other and communicate in situation dependant ways. This new era of connected manufacturing has brought about new requirements for machines which are being deployed to the manufacturing shop floor. Cyber physical machines are not only required to execute industrial or manufacturing process, but they need to be capable of meeting other requirements, such as self-diagnoses, intelligent decision making, modularity and networkability. This research explores the design process of cyber physical machines as it is applied to the design of an industrial robotic manipulator.

Keywords : Industry 4.0; Design Process; Manufacturing

Abbreviations : CPPS: Cyber Physical Production Systems; CPM: Cyber Physical Machines; IoT: Internet of Things; CPS: Cyber Physical Systems; FDM: Fused Deposition Modelling, SLA: Stereo lithography; DP: Design Parameters; FR: Functional Requirements; COTS: Commercially Off The Shelf

\section{Introduction}

\section{Cyber physical production systems}

The "Internet of Things (IoT)" and "Cyber Physical Systems (CPS)" [1] are bringing about a digital transformation to the traditional shop floor. The full industrial implementation of Cyber Physical Production Systems(CPPS) in manufacturing environments is bringing about the fourth industrial revolution or as defined by the German Federal Minister of Education and Research, Industry 4.0 [2].

Cyber Physical Production Systems (CPPS) consist of autonomous and cooperative elements (e.g. Cyber Physical Machines) and systems (e.g. Smart Factories) that are connected with each other in situation dependent ways, on and across all levels of production, from the processes level up to factory and production levels [3]. This new reality is being brought about by advances in technologies such as low power electronics, wireless communications, smart sensors [4], cloud manufacturing [5], advanced communication protocols such as OPC/UA [6] and data models such as Automation ML [7].

\section{Cyber physical machines}

As previously stated CPPS are composed of smart machines which are networked to each other.Smart machines, or cyber physical machines, are at the core of Industry 4.0. They are embedded with new capabilities and enabling technologies. This makes it possible to achieve functions which were not previously possible. For example, sensors on board of a cyber physical machine can be monitored remotely in real-time. This allows an intelligent Condition Monitoring System (CMS) to diagnose the machines condition and make it possible to carry out routine maintenance before a breakdown in the machine, possibly avoiding costly downtime [8].

The need for continuous adaptation has also driven the development of approaches that implement the concept of plugand-produce. Plug-and-produce allows for different elements of a production system to be added and removed from the production system depending on the needs of production. This concept of plug-and-produce also allows for the development of modular production systems. As explained by several authors, Schleipen et al. [9], Onori [10] and Maeda [11], the concept of plug-and-produce must be supported not only from a mechanical function, but also by the development of new and improved software and control paradigms.

Therefore another characteristic which is central to CPPS is decentralized and cognitive control. This is achieved by using machines that have embedded processing and networking capabilities [12]. This distributed control is gaining further 
popularity with the capability to use cognitive processing to analyze data gathered from machine sensors which allows for the decentralization of the CPPS control.

\section{Collaborative and connected robotics}

.The emergence of CPPSs has also brought with it an increased utilization of robotics. This trend has been highlighted at the World Economic Forum in Davos, which identifies advanced robotics as one of the main technological drivers behind Industry 4.0. Industrial robotics provides high efficiency and precision whilst being flexible and redeployable. Robots have seen increased utilization where fast cycle times of repetitive tasks needs to be maintained.

That said, this does not mean that humans will be completely eradicated from the shop floor. In fact based on detailed studies and experimentation conducted, Pfeiffer [13] argues that human experience will be still needed on the future shop floor. Based on this, the need for humans and robots to collaborate together on manufacturing operations will increase in the coming years [14]. In response to this growing need, it can in fact be seen how all major robot manufacturers are introducing to their lineup collaborative robots who are capable of working hand-in-hand with human operators. Collaborative robots are industrial robots which have been designed to confirm with specific safety standards which allow them to work within close proximity to human operators.

\section{Research aims}

Cyber physical machines need to be developed with specific characteristics as described above, in order to be implemented into CPPSs. These include characteristics such as communication, intelligence, modularity, which would allow these robots to communicate with the manufacturing cloud. The first activity in designing cyber physical machines is to define the requirements that need to be met and the functions which need to be executed. A solution is then synthesised from a set of design elements in order to meet these requirements. As will be presented in Section 2 from a review of the state of the art there are very few approaches which tackle the design approach of cyber physical machines.In order to understand the design process of cyber physical machines this research aims to explore the design process of a cyber physical industrial robotic manipulator.Section 3therefore describes the design process utilized to develop the industrial robotic manipulator, and the knowledge which was gained from this exercise. Section 4 then presents the prototype design and implementation. The conclusions and future work relating to this research are presented in Section 5.

\section{State of the Art}

In this section the authors present the state of the art relating to the topics of this research. Networked robots are defined as a system of multiple robots communicating with each other over a network, thus coordinating various tasks and roles, sometimes even with human operators. This means that networked robots are classified into two classes: teleoperated and autonomous. Teleoperated robots are controlled by humans, who send commands and receive feedback over the network. Autonomous robots communicate together as mentioned earlier, without the necessity of human intervention [15]. For autonomous robotic networks, it is ideal to have a decentralised network, thus allowing for a set of industrial robots to work together over a network. This promotes fast and optimum communication between these robots $[15,16]$ without the need of other robot systems to receive the information from a centralised controller.

Democratization of technology refers to the process by which more people rapidly gain access to technology. An approach to meet this need is to facilitate the implementation of robotics by developing cheaper and more customizable robots that can be easily implemented by small to medium enterprises. Rapid prototyping technology is thereforeoften used to develop open-source 3D printable dextrous anthropomorphic robots. By using rapid prototyping, the designers were able to create the design at a low cost. Moreover, the hardware and software designs were made available online, thus encouraging further improvement of the concept by the engineering community. This introduces an activity known as the democratisation of robotics, which means that the design of this robot system had to be accessible and usable by anyone $[17,18]$. Onal et al. have similarly succeeded in using rapid prototyping to provide a quick and inexpensive method to produce robotic manipulators [19]. In the case of Onal et al., 3D printing methods were used to create robot designs based on the structure of origami. Although this design is not currently used in any industrial applications, it proves the possibility of using RP to reduce costs while maintaining quality and increasing production speed $[18,19]$. We can note that several enterprises are founded on the basis of rapid prototy ping robotic manipulators such as Franka Emika, which has created its own RP robotic manipulator. This has seven degrees of freedom, and is also able to communicate using Ethernet (TCP/IP) network standards. This manipulator also incorporates several safety features, such as collision detection, force sensing and virtual wall collision avoidance [20].

During this research several design methods for CPPS and CPS were also reviewed [21-23]. Whilst they all highlight the multiple perspectives of CPPS, none of these illustrates the design process from requirements to the final design. This lack of CPPS design methods has also been highlighted by Fisher et al. [23].

From the literature review carried out the authors can conclude that there is no approach which combines 3D printed robots designed using a generative design approach 
for use in CPPS. As argued in Section 1, such an approach would decrease implementation costs and therefore sustain the democratization of robotics hence supporting SMEs in implementing CPPS.

\section{Cyber Physical Machine Design Process}

The design process must describe the design activities from goal (the requirements) to means (the approved design). To meet the aims of cyber physical production, a design process for Cyber Physical Machines (CPM) needs to be utilized that that takes into consideration not only the Physical but also the Cyber perspectives. This research utilized the systematic design process depicted by Roozenburg's basic design cycle [24] as a basis to explore the activities involved in designing CPMs. The different activities involved in designing CPMs being proposed by this approach will therefore be described in the next section.

\section{Analysis of CPM functional requirements}

The first activity carried out in design is an Analysis of the requirements that need to be met. When starting a new design cycle the designer begins by analyzing the requirements for the CPM. During the analysis the designer forms a better understanding of the problems (problem statement) and determines the goals that need to be achieved.
The basic goal of a CPM is to carry out the required manufacturing process at the required time, cost and quality and flexibility [25]. The analysis of the CPM requirements will also define the criteria by which the manufacturing system solution will be evaluated in future design activities. As described in the introduction, beyond these basic requirements, there are several other requirements which need to be met by CPMs. Therefore, if a CPM needs to operate within a CPPS the CPM requirements must include the capabilities of configurability, modularity, diagnosability and connectability. If the CPM solution does not meet these requirements it cannot be considered as a cyber physical system.

\section{CPM synthesis}

The next activity in the design cycle is Synthesis. Synthesis can be defined as the combination of components or elements to form a connected whole. It is in this activity of the design process that the CPM designer develops solutions for the design problems. Blessing [26] states that the synthesis activity can be broken down into some sub-activities. For example the 'generate' activity creates or finds elements suitable for the solution which are then 'selected' and 'synthesised' into possible solutions. It is also important to note that designers make selection commitments based on the CPM requirements defined in the previous activity.

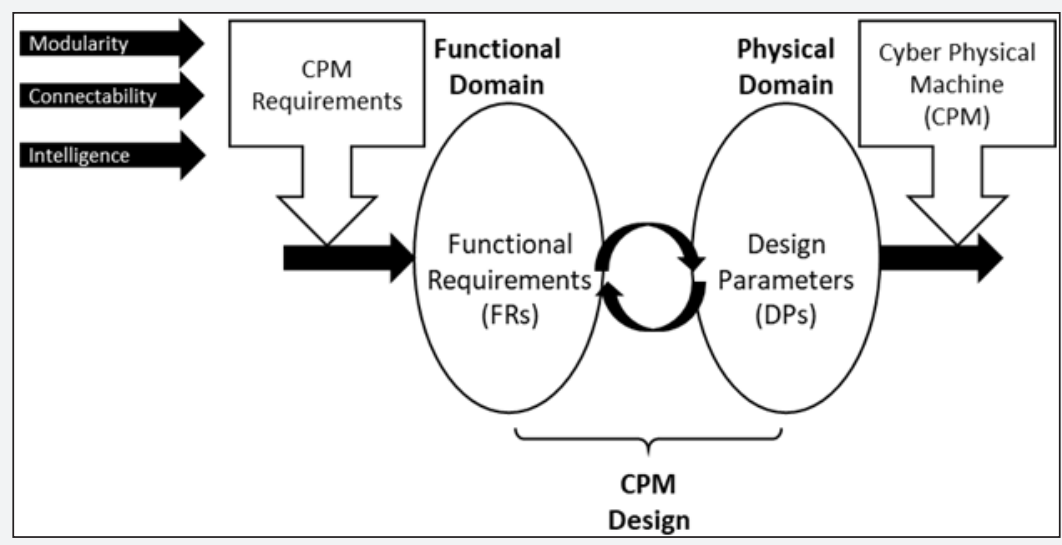

Figure 1: Unidirectional design framework, adapted from [29].

Based on the principles of axiomatic design [27], and as illustrated in Figure 1, Chryssolouris [25] and ElMaraghy [28] discuss that design synthesis is the activity of mapping from the functional requirements (FRs) onto suitable values of design parameters (DPs).

These decision variables combine to make a system, which describes both the physical design (Compositional View) and the manner of operation of the CPM (Functional View) [29].

This research proposes that during synthesis of CPM, consciously or not, designers make commitment in the cyber and physical domains. This research is therefore prescribing a synthesis design approach were designers take provisional commitments in these two domains. The result of the synthesis from the different domains of CPM design is a provisional design solution.

Simulation: The next stage of the CPM design cycle involves the Simulation of the provisional CPM design solution. Simulation involves the generation of an artificial history of a system and the observation of that history to draw inferences concerning the operating characteristics of the real system. The result of this study is the expected properties of the provisional CPM design solution. As explained by Hehenberger et al. [30], in order to simulate the full capabilities of a CPM, tools are required which model and simulate not only the 


\section{Robotics \& Automation Engineering Journal}

physical performance of the CPM, but also the information and communications exchange between systems.

Evaluation: During Evaluation of the provisional design solution the expected properties are compared to the design criteria established during the analysis stage. A value is then given to that design solution to quantify how well the provisional solution meets the CPM requirements.

Decision: The manufacturing system designer will then Decide whether to continue developing the design by further elaborating the provisional design or whether to try a different type of solution to generate a better design proposal. Once the manufacturing system designer is satisfied that the provisional design meets the requirements and criteria then the status will be upgraded to that of final design and the project can move on to implementation planning.

\section{Prototype Design and Implementation}

The CPM design process previously discussed was used during this research in order to design and implement the cyber connected industrial robotic manipulator which is discussed in this section.

\section{Physical Component Design}

The physical component design process results in the physical interface of the robotic manipulator. The physical system design utilizes a set of robotic joint modules, some of which are connected by a link module. Once produced and assembled, the robotic manipulator is essentially an articulated robot as shown in Figure 2. The manipulator is driven with the use of stepper motors in the joint modules.

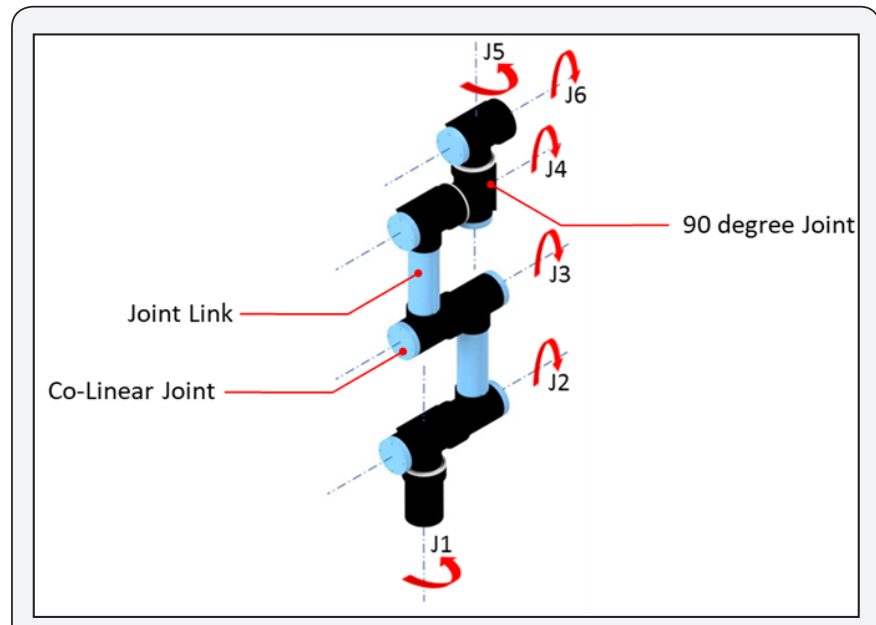

Figure 2: Physical model of the industrial robotic manipulator.

Design for modularity: In order to meet the requirements of being customizable for different industrial applications, the physical robotic manipulator design is configured by selecting, adding and removing joint and link modules. This design configuration was chosen specifically as it allows for the design of a modular industrial robotic manipulator, with the addition or subtraction of joints and motors depending on the application required. The design for modularity approach adopted here means that modules and interfaces between modules needed to be designed. The main module of this robotic manipulator is the joint which contains the servo motor. Two different configurations of this module were developed; the co-linear axis of rotation module, and the 90-degree axis of rotation module. The other module which was designed was the joint link module which connects the joints to each other.

The main configuration of the robotic manipulator is the one illustrated in Figure 2, and which employs six degrees of freedom. This means that the robotic manipulator is made up of six joints, each of which is a revolute joint. The six degrees of freedom ensure that the end effectors can reach any position and orientation within the workspace of the manipulator. Different stepper motor sizes and gear ratios are used in order to lift a $1.5 \mathrm{~kg}$ load at maximum extension.

Design for 3D printing: Since this robotic manipulator was intended to be mostly rapid prototyped, several factors and constraints needed to be taken into account during production of the prototype. Fused Deposition Modelling (FDM) and Stereo lithography (SLA) were used as the processes of rapid prototyping. The process was chosen based on the geometry of each part. Parts which had important geometry on one of their sides were 3D printed using SLA, such as the specially designed gears. Placing the side with the important geometry away from the support material was important for the preservation of said geometry.

On the other hand, parts which have a flat surface on one of their sides were printed using FDM, with that side being placed on the printing bed. The use of FDM was also encouraged when 3D printed parts required support material in internal and unreachable parts. Soluble support was used in these cases, making the support material easily removable during postprocessing.

In designing for 3D printing, an important factor considered was the minimization of weight. While modeling using CAD, several parts were designed to have the minimum wall thickness possible. This first step led to the search for other possible means of weight reduction. This included reduction in the diameter of the joints modules which led to all the internal parts also being smaller, and thus lighter.

\section{Cyber component design}

Requirements: The design goal for the control system of this device is for it to encourage integration with other systems and to encourage further development by system integrators. Integration must be done in a simple and rapid manner, on both the hardware and software domains. The system must be based on standardized or open source software control 
and development tools. To maintain a democratic design, the control hardware must be based on commercially off the shelf parts (COTS), enabling a low cost solution.

Industrial Ethernet: An industrial network based on Ethernet hardware shall serve as the backbone of the control system. There are multiple relevant industrial Ethernet protocols; the one selected for this solution is Ether CAT.

Ether CAT is an industrial network based on the standard Ethernet physical layer (Ethernet PHY). The network is deterministic, and can be used in a real-time environment. It supports shorter cycle times than other industrial networks, which make it suitable for motion control applications. Apart from the suitable performance Ether CAT provides, there are other factors which make it suitable for a system intended for the democratization of robotic technologies.

When networks are based on the Ethernet PHY, they would typically require a managed network switch, every Ether CAT device has two Ethernet PHYs which enable them to be connected via a daisy chain configuration, eliminating the need for a network switch if a ring network topology suffices. Furthermore, Ether CAT slave devices are responsible for controlling the timing of the network, without the need for a specialized master network controller. Therefore, the master device in an Ether CAT network can use common COTS Ethernet network interface cards (NIC), keeping the cost of implementing an Ether CAT network even lower. The use of a standard NIC allows for the implementation of an Ether CAT master through the use of any suitable device with basic Ethernet capabilities, this includes using a Raspberry Pi should the developer see fit.

Ether CAT is an open source technology, encouraging the development of hardware and software to work with this technology.

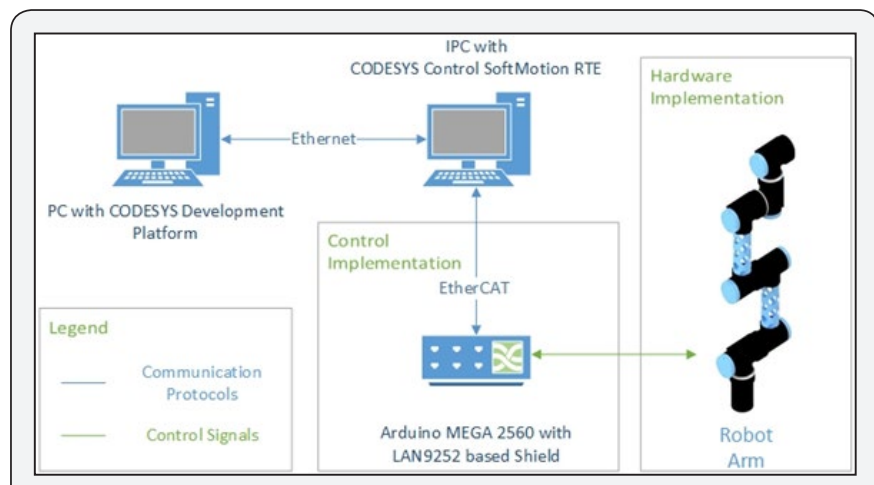

Figure 3 : Robotic Manipulator Control.

Robot controller: As illustrated in Figure 3, the robot controller was based on the ATMega2560, developed through Arduino/Genuino development platforms. Apart from the low cost required for developing solutions with Arduino, the vast user base contributing to various projects shortens and simplifies the microcontroller development cycle.

Asillustrated in Figure 3, the microcontroller was interfaced to the Ether CAT network via Microchip's LAN9252 Ether CAT controller for slave devices using SPI communication. A COTS add-on (shield) to the Arduino platform based on the LAN9252 is produced by $A B \& T$ Srl. The robot controller receives the joint positional data from its Ether CAT master and subsequently translates from joint position to the required pulses to drive the stepper motor.

Master controller: To support the flexibility required in the control system to adapt to different robot kinematic parameters, a real-time soft PLC was used. The soft PLC implemented was CODESYS Control Soft Motion RTE, it is developed using the IEC 61131-3 compliant CODESYS V3.5 development environment. The CODESYS development environment is free of charge, it also includes non-real-time versions of the soft PLC systems.

Once again, the low cost of the system enables the democratization of the control software and hardware. As of the time of writing, the real-time versions of the soft PLC systems can be downloaded and used without a license for a limited time before requiring a restart. CODESYS Soft Motion also has the inbuilt functionality to derive robot kinematics by defining the robot's Denavit-Hartenberg parameters (DH parameters).

\section{Conclusion}

This research therefore explored the design process of Cyber Physical Machines, through the application of this process to an industrial robotic manipulator. This approach considers not only the physical and hardware development but also the cyber and connectivity perspectives of CPMs. Future work by this research initiative will explore in further detail the cyber and physical elements of CPMs. The aim is to develop a knowledge based design support tool which can guide and support designers in taking decisions during the CPM design process.

\section{Acknowledgement}

The authors acknowledge the University of Malta for the financial support through the Research Grant "Digital Planning and Simulation for the Factory of the Future" (Vote No. IMERP 05-16).

\section{References}

1. Lee EA, Seshia S (2015) Introduction to Embedded Systems - A CyberPhysical Systems Approach. Second Edition. UC Berkeley.

2. Lasi H, Fettke P, Kemper HG, Feld T, Hoffmann M (2014) Industry 4.0. Bus Inf Syst Eng 6(4): 239-42.

3. Monostori L (2014) Cyber-physical Production Systems: Roots, Expectations and R\&D Challenges. Procedia CIRP 17: 9-13. 


\section{Robotics \& Automation Engineering Journal}

4. Vadde S, Kamarthi SV, Berry NM (2005) Smart sensors in a flow-line manufacturing system: an agent-based simulation. Int Conf Integr Knowl. Intensive Multi-Agent Syst. 2005 pp. 635-640.

5. Xu X (2012) From cloud computing to cloud manufacturing. Robot Comput-Integr Manuf 28(1): 75-86.

6. IEC 62541. OPC Unified Architecture, V2.0 2016.

7. Drath R, Luder A, Peschke J, Hundt L (2008) Automation ML - the glue for seamless automation engineering. 2008 IEEE Int Conf Emerg Technol Fact Autom, Pp. 616-23.

8. Ferreiro S, Konde E, Fernández S, Prado A (2016) Industry 4.0: Predictive Intelligent Maintenance for Production Equipment. Conf Third Eur Conf Progn Health Manag Soc, Bilbao, Spain.

9. Schleipen M, Lüder A, Sauer O, et al. (2015) Requirements and concept for Plug-and-Work: Adaptivity in the context of Industry 4.0. Autom AT 63: 801-820.

10. Onori M, Lohse N, Barata J, Hanisch C (2012) The IDEAS project: plug \& produce at shop-floor level. Assem Autom 32(2): 124-34.

11. Yusuke Maeda, Haruka Kikuchi, Hidemitsu Izawa, Hiroki Ogawa, Masao Sugi, Tamio Arai (2007) "Plug \& Produce" functions for an easily reconfigurable robotic assembly cell. Assem Autom 27: 253-60.

12. Verl A, Lechler A, Schlechtendahl J (2012) Glocalized cyber physical production systems. Prod Eng 6(6): 643-649.

13. Pfeiffer S (2016) Robots, Industry 4.0 and Humans, or Why Assembly Work Is More than Routine Work. Societies 6(2): 16

14. Richard Bloss (2016) Collaborative robots are rapidly providing major improvements in productivity, safety, programing ease, portability and cost while addressing many new applications. Ind Robot Int J 43: 463 468.

15. Siciliano B, Khatib O (2008) Springer Handbook of Robotics. Springer Science \& Business Media.

16. Schwager M, Rus D, Slotine JJ (2009) Decentralized, Adaptive Coverage Control for Networked Robots. Int J Robot Res 28(3): 357-75.

17. Bulgarelli A, Toscana G, Russo LO, Bona B (2016) A Low-Cost Open Source 3D-Printable Dexterous Anthropomorphic Robotic Hand with

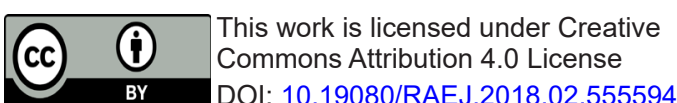

Commons Attribution 4.0 License

DOI: 10.19080/RAEJ.2018.02.555594 a Parallel Spherical Joint Wrist for Sign Languages Reproduction. Int J Adv Robot Syst 13:1.

18. Francalanza E, Fenech A, Cutajar P (2017) Generative design in the Development of a Robotic Manipulator, Gulf of Naples, Italy: Elsevier BV.

19. Onal CD, Wood RJ, Rus D (2017) Towards Printable Robotics: OrigamiInspired Planar Fabrication of Three-Dimensional Mechanisms 2011.

20. FRANKA EMIKA | (accessed May 9, 2017).

21. Khalid A, Kirisci P, Ghrairi Z, Thoben K-D, Pannek J (2016) A methodology to develop collaborative robotic cyber physical systems for production environments. Logist Res 9: 23.

22. Lee J, Bagheri B, Kao HA (2015) A Cyber-Physical Systems architecture for Industry 4.0-based manufacturing systems. Manuf Lett 3: 18-23.

23. Fisher A, Jacobson CA, Lee EA, Murray RM, Sangiovanni-Vincentelli A, et al. (2014) Industrial Cyber-Physical Systems - iCyPhy. In: Aiguier M, Boulanger F, Krob D, Marchal C (eds,). Complex Syst Des Manag. Springer International Publishing, p. 21-37.

24. Roozenburg NFM, Eekels J (1995) Product Design: Fundamentals and Methods. John Wiley \& Sons.

25. Chryssolouris G (2006) Manufacturing Systems: Theory and Practice.

26. Blessing L (1994) A Process-Based Approach To Computer Supported Engineering Design.

27. Suh NP (2001) Axiomatic Design: Advances and Applications. Oxford University Press, USA.

28. AlGeddawy T, ElMaraghy H (2011) Manufacturing systems synthesis using knowledge discovery. CIRP Ann - Manuf Technol 60: 437-440.

29. AlGeddawy TN, ElMaraghy HA (2009) Changeability Effect on Manufacturing Systems Design. In: ElMaraghy HA, editor. Chang. Reconfigurable Manuf Syst, London: Springer London; n.d, p. 267-83.

30. Hehenberger P, Vogel-Heuser B, Bradley D, Eynard B, Tomiyama T, et al. (2016) Design, modelling, simulation and integration of cyber physical systems: Methods and applications. Comput Ind 82: 273-89.

\section{Your next submission with Juniper Publishers will reach you the below assets}

- Quality Editorial service

- Swift Peer Review

- Reprints availability

- E-prints Service

- Manuscript Podcast for convenient understanding

- Global attainment for your research

- Manuscript accessibility in different formats

( Pdf, E-pub, Full Text, Audio)

- Unceasing customer service

Track the below URL for one-step submission https://juniperpublishers.com/online-submission.php 